\title{
Maternal Mortality at the Teaching Hospital of Mother and Child Lagoon (CHU-MEL) in Benin: A Preventable Drama?
}

\author{
M. Aboubakar ${ }^{*}$, J. Akodjenou${ }^{1}$, C. Echoudina' ${ }^{2}$, E. Ahounou², C. O. A. Biaou ${ }^{3}$, E. Zoumenou² \\ ${ }^{1}$ Teaching Hospital of Mother and Child Lagoon, Cotonou, Bénin \\ ${ }^{2}$ National and Teaching Hospital, Hubert Koutoukou Maga, Cotonou, Bénin \\ ${ }^{3}$ Régional Institute of Public Health, Ouidah, Benin \\ Email: *moufaliloua@yahoo.fr
}

How to cite this paper: Aboubakar, M. Akodjenou, J., Echoudina, C., Ahounou, E., Biaou, C.O.A. and Zoumenou, E. (2021) Maternal Mortality at the Teaching Hospital of Mother and Child Lagoon (CHUMEL) in Benin: A Preventable Drama? Open Journal of Obstetrics and Gynecology, 11, 315-325.

https://doi.org/10.4236/ojog.2021.113032

Received: January 27, 2021

Accepted: March 26, 2021

Published: March 29, 2021

Copyright (c) 2021 by author(s) and Scientific Research Publishing Inc. This work is licensed under the Creative Commons Attribution International License (CC BY 4.0).

http://creativecommons.org/licenses/by/4.0/

\begin{abstract}
Introduction: The maternal mortality ratio in developing countries is 239/ 100,000 live births (LV) in 2015, compared to $12 / 100,000$ live births (LV) in developed countries. This study aims to analyze the avoidability of maternal deaths at the CHU-MEL from 2015 to 2019. Patients and Method: This was a descriptive analytical study with retrospective data collection from January 1st 2015 to August 31st 2019, i.e. 56 months. The data were collected from medical records, maternal death registers, anaesthesia registers. They were entered and analyzed using Epi info version 7 software. The associations between avoidability of death and aetiologies were tested using $\mathrm{Chi}^{2}$ or Fisher's test as appropriate. The threshold for statistical significance was $5 \%$. Results: The maternal mortality ratio over 5 years was 905 maternal deaths per 100,000 $\mathrm{LV}$. The age of the deceased women ranged from 15 to 44 years, with an average of $29.09 \pm 7.04$ years. They were illiterate or primary educated $(47.02 \%)$, married $(64.50 \%)$ primigravida or paucigravidae $(47.02 \%)$ and primiparous or pauciparous (59.52\%). Of the 151 maternal deaths, (90.73\%) were deemed preventable. Delay was the main reason for maternal death, and $82.11 \%$ had at least one type of delay. There was no significant difference between the avoidability of death and the main aetiologies of haemorrhage $(p=0.865)$, infections $(\mathrm{p}=0.208)$ and hypertensive complications $(\mathrm{p}=0.438)$. Conclusion: The maternal mortality ratio during the study period was 905 maternal deaths per 100,000 LV. Deaths were preventable in $90.73 \%$ of cases. The avoidability factors found were varied.
\end{abstract}

\section{Keywords}

Maternal Mortality, Avoidability, CHU-MEL, Benin 


\section{Introduction}

The mortality rate is an indicator of the quality of a country's health system. Maternal mortality provides information on the risk incurred by women during pregnancy and childbirth. This risk is a reflection of the defiency and strengths of the functioning of the health system.

The maternal morality ratio in developing countries is $239 / 100,000$ live births (LV) in 2015, compared to 12/100,000 live births in developed countries [1].

The probability that a young woman will one day die from a cause related to pregnancy or childbirth is 1 in 4900 in developed countries, compared to 1 in 180 in developing countries [1]. In Africa, the risk of maternal mortality is 1 in 16 women, a consequence of the collapse of health systems [2].

In Benin, maternal mortality is 410 deaths per 100,000 live births in 2011 [3] and 391 deaths per 100,000 live births in 2018 [4]. This decrease is not significant despite government efforts to provide free caesarean sections and improve the health system [3]. To reduce the maternal death rate, it is necessary to identify the barriers that limit access to quality maternal health care and to take action to address modifiable causes at all levels of the health system. The objective of this work is to study maternal deaths in order to assess their avoidability at a reference maternity in Cotonou.

\section{Patients and Method}

We carried out a descriptive analytical study with retrospective data collection from 1st January 2015 to 31 August 2019, i.e. a duration of 56 months. The study population consisted of maternal death records at the CHUMEL. Maternal death was considered to be "the death of a woman occurring during pregnancy or within 42 days of its termination, whatever the duration or location, for any cause determined or aggravated by the pregnancy or the care it caused, but neither accidental nor fortuitous". We have exhaustively included records of maternal deaths that occurred during the study period.

A maternal death is "preventable" or "possibly preventable" if one or more changes in the patient's care pathway could have changed the fatal outcome.

The avoidability factors involved were classified into three categories:

- related to the adequacy of care, (adapted therapy, adapted but insufficient therapy, unsuitable therapy, therapeutic errors

- related to the organization of care, (delay in care, reception, poor supervision)

- related to the patient's interaction with the health care system (refusal of treatment, notion of discharge against medical advice etc.).

- Death was avoidable when at least one of the criteria exists.

The death was deemed preventable if at least one of the criteria was met.

The independent variables were socio-demographic, relating to gynaecological-obstetrical history, medical and surgical history, clinical data, and therapeutic management. 
The data was collected using pre-tested forms, by reviewing medical records, maternal death records, anaesthesia records, operating protocols and maternal death reports.

The completeness and consistency of the data collected was checked at the end of the day to ensure the quality of the data collected. The data was then entered and analysed using Epi info version 7 software. The associations between the avoidability of death and aetiologies were tested using $\mathrm{Chi}^{2}$ or Fisher's test as appropriate. The strength of the associations was measured through raw Odds Ratios (OR) and their 95\% confidence interval [95\% CI]. The threshold for statistical significance was $5 \%$.

\section{Results}

\section{Frequency}

During the period of study, there were 161 cases of maternal deaths, of which we excluded 10 cases from this study ( 5 cases of unrecovered records, 3 cases of death before admission and 2 cases of death on admission). During the same period, CHU-MEL registered 18,611 deliveries and 17,781 live births. The Maternal Mortality Ratio over this period is 905 maternal deaths per 100,000 NV.

\section{Evolution of maternal deaths from 2015 to 2019}

There was an increasing trend in the maternal mortality ratio in CHU-MEL from 2015 to 2019 (Table 1).

\section{Socio-demographic characteristics of deceased patients}

The ages of the deceased patients ranged from 15 to 44 years. The mean age was $29.09 \pm 7.04$ years and the median age was 29 years (IIQ: 23 - 36). Patients under 35 years of age represented $65.23 \%$ of the population (Table 2).

Of the 151 women, 71 (47.02\%) had not reached secondary school or were illiterate.

\section{Gynaecological-obstetrical history}

Deceased patients were primigravida or paucigravidain $47.02 \%$ of cases; primiparous or pauciparous in $59.52 \%$ of cases.

Previous pregnancies medical History

The main medical history found in previous pregnancies were caesarean section

Table 1. Parameters for the study of maternal deaths at CHUMEL from 2015 to 2019.

\begin{tabular}{ccccc}
\hline Year & Deliveries & Live birth & Maternal death & Mortality Ratio $^{* *}$ \\
\hline 2015 & 3609 & 3368 & 20 & 594 \\
2016 & 4060 & 3706 & 30 & 809 \\
2017 & 3296 & 3103 & 25 & 806 \\
2018 & 4377 & 4095 & 45 & 1099 \\
$2019^{*}$ & 3269 & 3509 & 41 & 1168 \\
$2015-2019$ & 18,611 & 17,781 & 161 & 905 \\
\hline
\end{tabular}

*From January to August; ${ }^{\star *}$ Per 100,000 Live births. 
Table 2. Socio-demographic characcteristics of the patients.

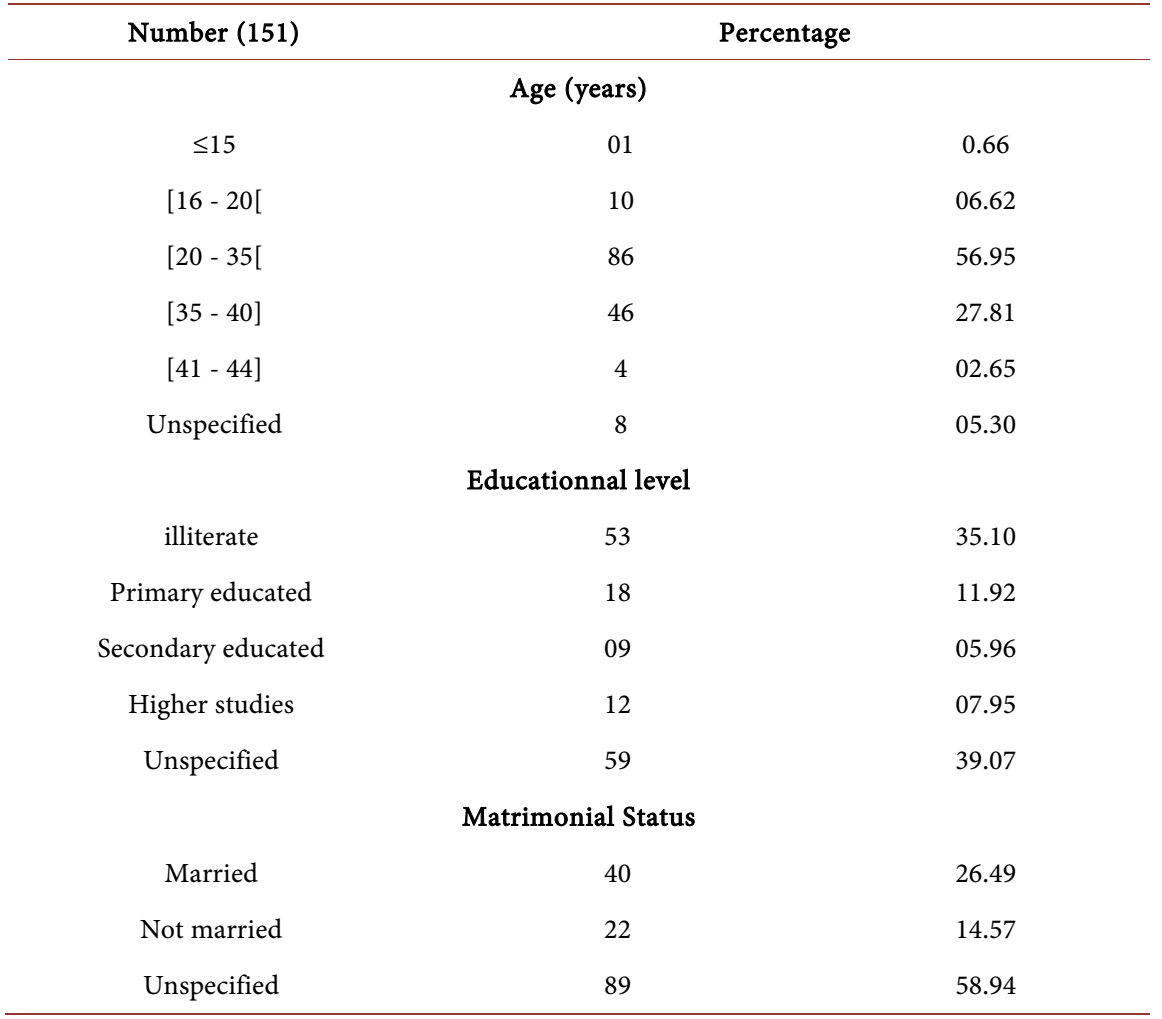

(22.76\%), hypertension and its complications (4.18\%), ectopic pregnancy (1.38\%) and placenta praevia $(0.69 \%)$.

\section{Pregnancy monitoring}

Information on the number of prenatal visit (PV) was only available in $18.54 \%$ of the cases and showed that 10 women did not attend any PV

In the majority of the deceased women $(92.02 \%)$ the pregnancy had not been followed or had been poorly followed. In $60.93 \%$ of the women, the minimum required prenatal check-up ${ }^{\star}$ had not been carried out.

${ }^{*}$ Blood grouping + rhesus factor (GSRH); blood count (CBC), albuminuria, hemoglobin electrophoresis, obstetrical ultrasound.

\section{Analysis of Avoidability \\ Origin of deceased women}

The women came from private $(38.41 \%)$ and public $(30.46 \%)$ health care and $22.51 \%$ came from traditional healers or from their homes.

\section{Reasons for referral}

A total of 125 women were admitted per referral. The main reason for referral was postpartum haemorrhage.

\section{Adequacy of treatment prior to referral}

Of the 125 women referred, $32(25.60 \%)$ had received care deemed appropriate before referral, i.e. in accordance with the protocols in force.

Referral conditions

Of the cases informed, $48.83 \%$ did not have a reliable intraveinous catether; 
45.61\% did not have a referral form, $82.29 \%$ had not benefited from medical transport and in $73.25 \%$ there was a delay between referral and arrival at the CHUMEL.

\section{Diagnostic and therapeutic errors}

Diagnostic errors were found in 8 women i.e. $5.30 \%$ of deceased women. Therapeutics were harmful in $5.30 \%(n=8 / 151)$ of these cases, we found 03 cases of acute pulmonary oedema (APO) due to iatrogenic overload.

\section{Inadequate and insufficient therapy}

Of the 151 women, 14 (9.27\%) were considered to be inadequately cared for, and $14(9.27 \%)$ were considered to be suitable, but $55.63 \%(\mathrm{n}=84 / 151)$ were considered to be unsuitable $(n=84 / 151)$.

\section{Poor follow-up}

Poor mother and foetal follow-up was noted in 58 women, i.e. $38.41 \%$ of the women who died.

\section{Types of delay}

A total of 124 women $(82.11 \%)$ had at least one type of delay. The third delay was the most frequent (63.58\%) (Table 3 ).

\section{Factors causin delay}

Several factors have been identified. The three main factors were, in order: lack of financial means $(n=78 / 124 ; 62.90 \%)$, lack of blood products $(n=59 / 124$; or $47.58 \%)$ and the unavailability of the operating room ( $\mathrm{n}=24$; or $19.35 \%)$ (Table 4).

\section{Avoidance of death}

Of the 151 maternal deaths, 137 (90.73\%) were deemed preventable (Figure 1).

Relationship between avoidability of death and main aetiologies

The analyses did not find a significant difference between the avoidability of death and the main aetiologies (Table 5).

Table 3. Distribution of maternal death by type of delay.

\begin{tabular}{ccc}
\hline & Effectif & $\%^{*}$ \\
\hline First delay & 53 & 35.10 \\
Yes & 80 & 52.98 \\
No & 18 & 11.92 \\
Not specified & & \\
Second delay & 51 & 33.77 \\
Yes & 82 & 54.30 \\
No & 18 & 11.92 \\
Not specified & & 63.58 \\
Third delay & 96 & 24.50 \\
Yes & 37 & 11.92 \\
No & 18 & \\
Not specified & &
\end{tabular}

*one woman could presents many delays. 
Table 4. Distribution of maternal deaths by delay factors*.

\begin{tabular}{ccc}
\hline & number & $\%$ \\
\hline Lack of financial means & 79 & 63.70 \\
Lack of blood products & 59 & 47.58 \\
Unavailabylity of operating room & 24 & 19.35 \\
Unavailabylity of transport means for reference & 6 & 4.84 \\
multiple Reference* & 3 & 2.42 \\
Childbirth at home & 2 & 1.61 \\
Lack of surgery materials & 1 & 0.81 \\
Lack of oxygen & 1 & 0.81 \\
\hline
\end{tabular}

${ }^{*}$ Several delay factors could be recorded for the same woman; ${ }^{*}$ Cases where patients are redirected between several health cares before the reference center.

Table 5. Relationship between avoidability of death and major aetiologies.

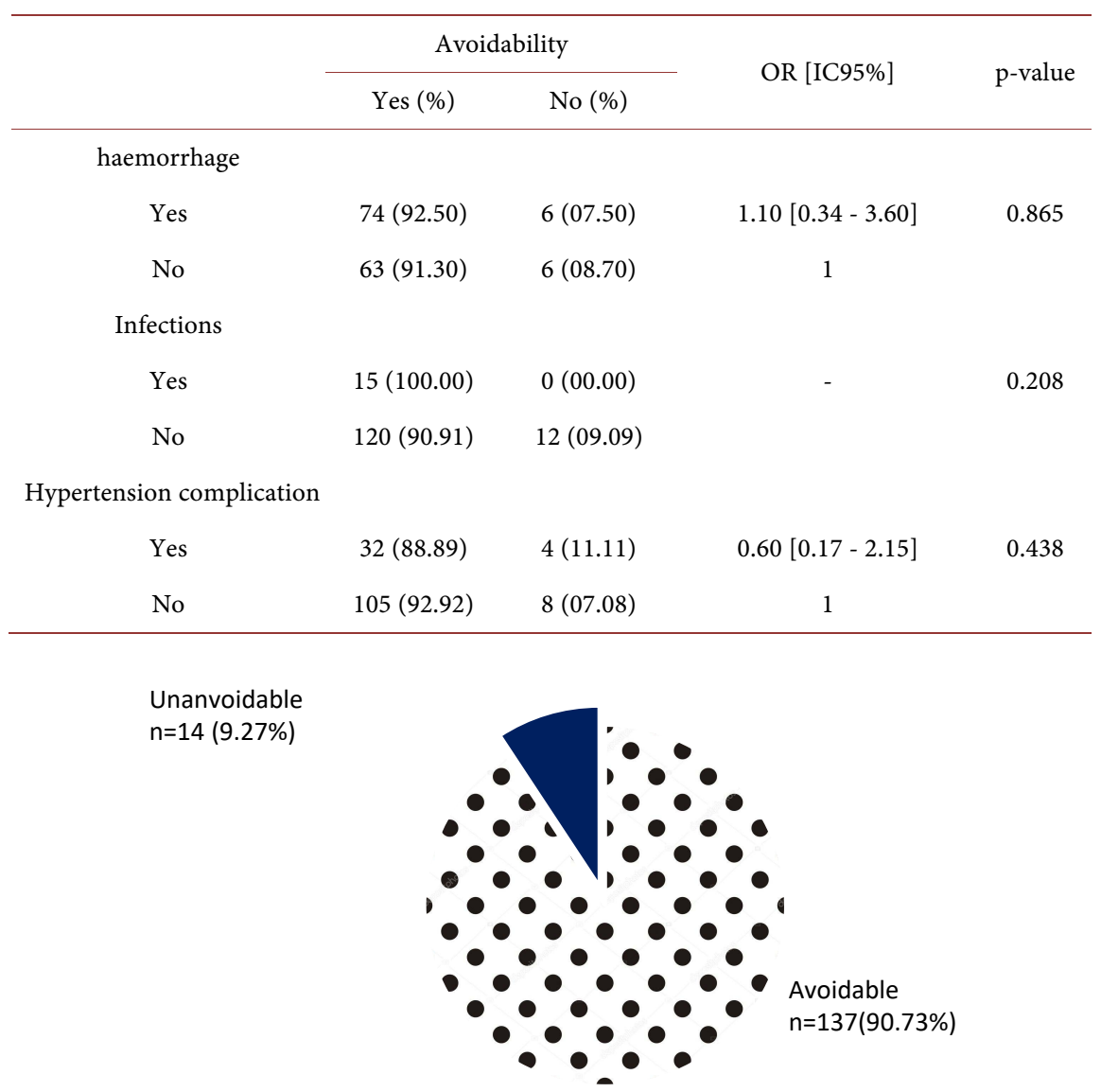

Figure 1. Distribution of deceased women according to avoidability of death.

\section{Discussion}

Our aim was to study the avoidability of maternal deaths at the CHU-MEL, to trace the relationships between maternal deaths and the different aetiologies. We therefore found that death was preventable in $90.37 \%(n=137 / 151)$ of cases and 
the reasons were both multiple and varied.

\section{Socio-demographic characteristics}

Age

The mean age of the deceased patients was $29.09 \pm 7.04$ years. The most affected age group was women between 20 and 35 years of age. Our results are comparable to those of Denakpo et al. at the same hospital who found in 2008 that the majority of women who died were between 20 and 29 years old [5]. Kane, in Mali, in 2016, also found in his study on audits of maternal deaths that the majority of women who died were in the age group of 20 - 35 years [6]. This can be explained by the fact that this age range matches with the age of optimal female fertility, with significant sexual activity. In Benin, according to DHS V, the highest fertility rates are observed among women aged 20 - 29 years [7].

The Confidential National Survey on Maternal Deaths (ENCMM) in France between 2010 and 2012 found that the majority of women who died were between 35 and 39 years of age [8]. This can be explained, on the one hand, by the preponderance of late pregnancies in Western societies and, on the other hand, by the existence of conditions such as diabetes and high blood pressure in old age which increase the risk of complications during pregnancy.

Level of education

Illiterate women and those with primary education were most represented, accounting for $47.02 \%$ of the population studied. This could be explained by the low enrolment rate for girls in developing countries and particularly in Benin [9]. Like us, Kane found in 2016 in his work at Referral Hospital IV (CSRIV) in the district of Bamako that illiterate women made up the majority of the population with a rate of $47 \%$ [6].

Marital status

Married women made up $64.5 \%$ of the deceased women in our study. Much higher figures were found by Kane at the CSRIV in the Bamako district, where $91 \%$ were married women [6]. This can be explained by the influence of the Muslim religion in this Muslim-majority country. Conversely, Foumane et al. found only $34.5 \%$ of the women who died at the Gynaeco-Obstetric and Paediatric Hospital in Yaoundé in 2010 to be married [10].

\section{Gynaecological-obstetrical history}

The pauciparous were the most represented in the population of women who died during the period (37.30\%). The same observation was made by Lefèvre in 2014 in a study in the public hospitals of Reunion Island with a rate of $44.4 \%$ of deceased pauciparous [11]. Fomulu et al. found in their study at the Teaching hospital of Yaoundé in 2006 noted that the majority of women who died were nulliparous (841 maternal deaths per 100,000 live births) and multiparous (600 maternal deaths per 100,000 live births). Both nulliparous and big multiparous women are populations at risk of developing complications during pregnancy [12]. Indeed, nulliparity is a pejorative factor through pregnancy toxemia and its major complication, eclampsia. Multiparity is responsible for the laxity of the 
uterine muscle, which favours vicious presentations, with a high risk of uterine rupture and postpartum haemorrhages.

\section{Avoidability analysis}

\section{Pregnancy monitoring}

The antenatal visit is the ideal time to ensure preventive measures such as screening for risk factors, tetanus vaccination, initiation of treatment and education of the woman about the course of pregnancy and childbirth [13]. In our study, $60.93 \%$ of the women had no pregnancy follow-up and $31.13 \%$ of the women had poor pregnancy follow-up. Kane in Bamako in 2014 found a much lower rate of $35 \%$ [6]. This situation is compatible with the low socio-economic and intellectual level of the population. Indeed, ignorance of the importance of antenatal care, the poor distribution and shortage of qualified human resources and the financial barrier are reasons for the low rate of prenatal visit in our developing countries.

\section{Terms of patients transferring}

The existence of a reliable intraveinous catether is an important element in the management of vital distress. It was absent in $48.83 \%$ of cases. Tshabu Aguemon et al. found a similar rate in a study carried out at maternity of Menontin hospital in Cotonou from 2007 to 2011 which showed that $46.86 \%$ of women who died had been referred without a intraveinous catether [14].

The transfer form, an important communication tool between the referring hospital and the referral hospital to ensure the continuum of care, was absent in $45.61 \%$ of cases. Other authors in Benin found rates ranging from 35\% to $75 \%$. [5] [14]. In $82.29 \%$ of cases, the transport was not medicalised (use of personal means, non-equipped ambulances, etc.), exposing the woman to a deterioration in her already very precarious condition during the journey. Other authors have found similar figures ranging from $78.6 \%$ to $80 \%$ [5] [14]. Many hospitals do not have ambulances. When they do exist, they are generally devoid of any resuscitation equipment and are only used to move the patient.

\section{Type of delay and factors of delay}

The first delay relates to late recourse to emergency care attributed to the family and the community: ignorance of danger signs, delay in taking a decision on the appeal, choice of type of care

The second delay relates to the problems of access to care facilities and reference, to the functioning of the referral system but also to the state of communication routes and means of transport: geographical access, means of transport, financial access, cultural acceptability of health services

The third delay relates to availability of quality services. It depends of the organisation and availability of health structures: availability of staff, availability of the technical platform, community perception of the quality of services provided

In our study, the majority of the deceased women (82.11\%) had experienced at least one type of delay. The most frequent type of delay was the third delay representing $63.58 \%$ of the cases. Baldé et al. also found in Guinea-Conakry in 
2012 that the 3rd delay was the most frequent, but with higher figures than ours. Indeed, they found that $87.5 \%$ of the women who died were victims of the third delay [15]. The main factors of loss of time identified in our study were lack of financial means (63.7\%), lack of blood products (47.58\%) and unavailability of the operating room (19.35\%). In Menontin hospital, Tshabu Aguemon et al. found no cases of lack of financial resources [14]. This can be explained by the fact that Menontin Hospital is a "first contact" referral hospital; patients go there with a good part of the available financial resources. However, they found a rate of unavailability of blood products comparable to that of our study. The same authors indicated that the unavailability of the operating room was noted in $39.53 \%$ of cases. This hospital has only one operating room shared between the obstetrics department and the other surgical specialties.

\section{Follow-up}

Poor follow-up of the mother and/or foetus may be due to poor organisation of care. Insufficient staff in the referral centers to cope with the various demands, but also under-equipment, are also factors that can explain this situation. We noted poor follow-up among $38.41 \%$ of the women. Tshabu Aguemon et al. found a lower rate in Menontin hospital (21\%) [14].

\section{Adequacy of therapy}

At the referring center

The quality of care before referral plays an important role in the prognosis of referred patients. However, many authors in Benin [14] and DRC [16] have found that more than $30 \%$ of patients did not receive adequate treatment at the referring centre. We noted a higher rate with $74.4 \%$ of patients not having received adequate treatment before referral.

The poor quality of care in the referring centers has multifactorial origins; one of the factors being the anarchic installation of many substandard health facilities that practice childbirth.

At the referal center

Among the inadequacies observed in the conduct here, appropriate but insufficient therapy (55.63\%) was the most frequent, far ahead of diagnostic errors (5.30\%), therapeutic errors (5.30\%) and inappropriate therapy (9.27\%). Tshabu Aguemon et al. found no diagnostic errors at Menontin Hospital. They reported that $25.83 \%$ of the patients were treated inadequately and in $20.93 \%$ the therapy was adequate but insufficient [14]. Denakpo et al. found at the CHUMEL in 2008 that the therapy was either inappropriate (20\% of cases) or insufficient ( $80 \%$ of cases) [5]. Bouvier-Colle found from 2010 to 2012 that $10 \%$ of maternal deaths in West Africa were due to therapeutic errors [17]. These inadequacies can be explained by the inadequacy of the technical platform, the insufficient number of health workers recruited for the task, and insufficient training and retraining on the new protocols in force.

\section{Avoidability itself}

While maternal mortality is an important indicator of the quality of a coun- 
try's health system, the preventability of these deaths is even more important. In our study, we found that the majority of recorded maternal deaths were preventable (90.73\%). Already in 2008 in the same hospital, Denakpo et al. reported that $100 \%$ of the deaths assessed were preventable [5]. These rates can be explained by the failure of the organisation of care and also of the content of care, which is often insufficient when it is adapted. The situation is similar in many developing countries. For example, the avoidability rates found by Baldé et al. in Guinea Conakry, Lefèvre in Reunion Island, and Ahbibi in Marrakech were $87.5 \%, 85 \%$ and $70 \%$ respectively [13] [17]. In all these studies, as in our own, deaths from direct causes were the most preventable. Although the maternal mortality ratio is significantly lower in France, deaths from direct causes remain the most preventable. Deneux-thanaux found a rate of 66\% in France from 2010 to 2012 [18].

\section{Limitation of the study}

An essential limitation of our study is the absence of certain essential information in medical records, which constitutes an information bias.However our objectives have been reached.

\section{Conclusion}

Our avoidability study found that deaths were preventable in $90.73 \%$ of cases. The avoidability factors were dysfunctions related to the organisation of care and the content of care. At CHU-MEL, we noted diagnostic errors, therapeutic errors, inappropriate therapy, poor monitoring and delays in care.

\section{Conflicts of Interest}

The authors declare no conflicts of interest regarding the publication of this paper.

\section{References}

[1] World Health Organization (WHO) (2019) Maternal Mortality.

[2] United Nations Development Programme (UNDP) (2015) Improving Maternal Health, Where Do We Stand?

[3] World Health Organization (WHO) (2014) Targets and Strategies to End Preventable Maternal Deaths: A Consensus Statement.

[4] Barry, B. (2008) Maternal Mortality: Causes and Facilitating Factors Determined by Verbal Autopsy in Bakel Department. Research Master's thesis, Cheik Anta Diop University, Dakar.

[5] Denakpo, J.L., Zoumenou, E., Hounkpatin, B., Aguemon, C. and Lokossou, A. (2010) Impact of Clinical Audits on the Quality of Emergency Obstetric and Neonatal Care at the Hôpital de la Mère et de l'Enfant Lagune in Cotonou, Benin. Journal de la $S A G O, 11,13-17$

[6] Kane, A. (2018) Audit of Maternal Deaths at the Reference Health Centre in Commune IV of Bamako District. Doctorate in Medicine Thesis No. 18M49, Faculté de Médecine et d'Odonto-Stomatologie (FMOS), Bamako, 67 p.

[7] Institut National de la Statistique et de l'Analyse Economique (INSAE) (2019) De- 
mographic Health Survey in Benin, 2017-2018. 5th Edition, INSAE and ICF, Cotonou, Rockville.

[8] National Institute of Health and Medical Research (INSERM) (2017) Characteristics of Deceased Women. In: Les morts maternelles en France: Mieux comprendre pour mieux prévenir. 5 th Report of the Confidential National Survey on Maternal Deaths (ENCMM) 2010-2012, Santé Publique France, Saint-Maurice, 230 p.

[9] United Nations Development Programme (UNDP) (2016) Goal 4: Quality Education.

[10] Foumane, P., Dohbit, J.S., Ngo Um Meka, E., Nkada, N.M., et al. (2015) Etiologies of Maternal Mortality at the Gynaeco-Obstetric and Pediatric Hospital in Yaoundé: A Series of 58 Deaths. Health Sciences and Disease, 16, 1-5.

[11] Lefèvre, J. (2014) Epidemiology of Hospital Maternal Mortality. Observational Study Carried out in Reunion Island. Thesis for Doctorate in Medicine No. 76, U.F.R des sciences médicales, Bordeaux, 131.

[12] Fomulu, F.J., Ngassa, P.N., Nong, T., Nana, P. and Nkwabong, E. (2009) Maternal Mortality at the Maternity Hospital of the Centre Hospitalier et Universitaire de Yaoundé, Cameroon: 5-Year Retrospective Study (2002 to 2006). Health Sciences and Disease, 10, 1-6.

[13] World Health Organization (WHO) (2016) WHO Recommendations for Prenatal Care to Make Pregnancy: A Positive Experience.

[14] Tshabu Aguemon, C., Adisso, S., Hounkpatin, B., Obossou, A. and Bashibiririrwa, K. (2014) Identification of Dysfunctions in the Management of Cases of Maternal Mortality in the Maternity Ward of the Menontin Hospital in Cotonou. Ramur, 19, 10-13.

[15] Baldé, I.S., Baldé, O., Camara, M.K., Diallo, A., et al. (2016) Review of Maternal Deaths in the Gynaecology and Obstetrics Department of the Donka CHU National Hospital in Conakry (Guinea). Revue International des Sciences Médicales, 18, 99-103.

[16] Kahindo, J.B., Karemere, H. and Mitangala, P. (2018) Explanatory Factors for Maternal Deaths in Hospitals: A Study of Six Health Zones in Eastern Democratic Republic of Congo. International Journal of Innovation and Applied Studies, 23, 559-568.

[17] Ouédraogo, C. and Bouvier-Colle, M.-H. (2002) Maternal Health in Francophone Africa-Maternal Mortality in West Africa: How, How Many and Why? The Journal de Gynécologie Obstétrique et Biologie de la Reproduction, 31, 80-89.

[18] Deneux-Tharaux, C. and Saucedo, M. (2017) Epidemiology of Maternal Mortality in France, 2010-2012. Gynécologie Obstétrique Fertilité \& Sénologie, 45, S8-S21. https://doi.org/10.1016/j.gofs.2017.10.025 